\title{
Preface: The Behaviorally Informed Organization
}

In the year 2008, Richard Thaler and Cass Sunstein published a book, Nudge, which has revolutionized the way in which organizations all over the world think about using behavioral science in their operations, and in particular in designing their last-mile interfaces. The field of behavioral science had been around for decades. In the years before Nudge was published, an academic discipline that had come to be known as behavioral economics had begun to capture the imagination of policymakers, governments, and the lay public alike. This interest was fueled by a Nobel Prize in 2002 to Daniel Kahneman, and the publication of books such as Predictably Irrational by Dan Ariely. Nudge harnessed all of this growing interest in the field and made it practical and real for organizations. The book provided a large number of examples of how organizations could steer the behavior of end-users without the use of restrictions, incentives, or even traditional communication-based persuasion techniques. The word "nudge" started becoming part of the organizational lexicon. Shortly after the book was published, the UK government launched their first ever Behavioral Insights Team within the Cabinet Office. Since then, a large number of governments - and eventually businesses - have begun to appreciate the importance of behavioral insights (BI) in their operations. In the foreword to this book, Cass Sunstein introduces nudging, and provides examples of its use. 
In academia, researchers in behavioral science have long been studying human behaviors. A more recent movement within academic economics - behavioral economics - seeks to apply behavioral science to economic decision making. Practitioners often refer to the learning from these fields as behavioral insights (BI). We will use the term "BI" as the catchall term that represents both the science and the findings and insights that arise from it. However, authors of individual chapters might use the word "science" or "economics" to reflect which part of the collective their material draws from, as well as their own philosophy.

Despite this growing surge in interest in BI, it is our contention that the potential of behavioral insights to fundamentally change the way in which organizations operate and interact with their stakeholders is nowhere near to being fully harnessed. Given that organizations are fundamentally in the business of changing the behavior of their internal and external stakeholders, and given that we have a rich science of how behavior change is brought about, this gap is a bit of an enigma. We see two major challenges for our field in the next decade. First, we have had a lot of success with pilot projects but have not yet developed a framework for how these pilots can be scaled. Second, we need to understand how organizations can become better behaviorally informed. We believe that overcoming the second challenge will allow us to tackle the first. We - along with several other behavioral scientists and partner organizations - have put together an international partnership of scientists and practitioners with the goal of understanding how organizations can better harness behavioral insights (see biorgpartnership.com). Our partnership is housed in the Behavioural Economics in Action Research at Rotman (BEAR) research center at the University of Toronto.

We believe that the gap between the promise of behavioral science and the actual adoption of BI arises from three fundamental factors. First, there is a mismatch between the expertise of an academic researcher and the needs of a practitioner. In particular, behavioral scientists tend to make careers by developing expertise in a particular phenomenon - a researcher who studies how social forces influence behaviors would recommend overweight consumers to sign a public weight-loss petition, whereas another researcher who studies 
incentives would suggest using money to reward weight loss. The practitioner, on the other hand, is interested in the outcome without a specific preference for any one phenomenon. Further, researchers are typically not keen on mixing up interventions, because the scientific value of academic research comes from its preciseness in testing how a particular intervention drives a behavioral change. If a researcher were to choose between using an intervention to achieve a moderate outcome and a cocktail of interventions to achieve a better overall outcome, the researcher will likely choose the former. The practitioner, on the other hand, needs a cocktail of interventions that yields significant behavioral change, but this need is unmet because behavioral scientists rarely research the formula for such a cocktail mix. Second, much behavioral science is done in a laboratory, and participants make static (i.e., at that point in time) choices in relatively sterile environments. In contrast, behaviors in the real world are often complex (i.e., multiple determined) and dynamic (i.e., liable to change as a function of past decisions). Furthermore, the practitioner is often interested in behaviors that are guided by habit (as part of a routine); these behaviors lose their flavors once they are isolated from their routines and studied as "single-shot" behaviors in the lab. Therefore, such lab-based findings may not be particularly insightful for practitioners who are interested in understanding habitual behaviors. These divergences limit the adoption of BI in practice. Finally, academic research is conducted in environments with an academic culture that encourages experimentations and testing, while the realities in organizations often require the practitioner to make quick decisions and to look decisive, constraints that might preclude experimentation and testing.

Compounding these fundamental reasons is a misunderstanding of how the process of behavior-change interventions actually works. A reading of past BI successes can easily lead to the misunderstanding that the application of BI is linear and therefore straightforward. However, each success is only the tip of a proverbial iceberg that contains a lot of groundwork, iterations, and experimental failures. Indeed, while the field needs to share its successes, it also needs to highlight the fact that BI is not a simple off-the-shelf solution that is guaranteed to work. 
Our partnership was set up with the objective of helping every organization become behaviorally informed. In particular, it seeks to develop a science for using behavioral science by addressing some of the reasons underlying the gap between the promise of behavioral science and its adoption in organizations. What is a behaviorally informed organization (BIOrg)? It is an organization that (a) recognizes that its external and internal stakeholders are human (and not "econs" - agents that comply with the assumptions of utility maximization); (b) understands the importance of context; (c) designs for humans; (d) believes in testing, learning, and adapting; and (e) has the agility to update products, processes, and programs in response to learning from experiments. In general, a BIOrg is deeply committed to being human compliant in every possible way!

This edited volume represents the first output from this international partnership. The book is designed to reflect our conceptual thinking, outline some early results from the partnership and an agenda for research and practice, and provide roadmaps to help both practitioners and academics converge in the common quest of developing behaviorally informed organizations. The book is divided into four parts. In Part 1, "The Behaviorally Informed Organization," four chapters lay out an agenda for what such an organization should be and could be. In chapter 1, Soman talks about the science of using behavioral science by developing a brief history of the field of behavioral science, outlining organizational realities, and generating a research agenda to help develop BIOrgs. In chapter 2, Feng and colleagues further develop an understanding of organizational realities and outline what resources and capabilities organizations need to develop in order to be truly behaviorally informed. In particular, they develop the notion of the cost of experimentation and make the point that driving down the cost of experimentation is key in developing behaviorally informed organizations. In chapter 3, Vinski asks and answers the question, "Why should organizations even want to be behaviorally informed?"; and in chapter 4, O'Malley and Peters add to that question by further addressing why organizations might actively resist the need to be behaviorally informed.

Organizational settings provide existing tools and also additional complexities, and in Part 2, "Overarching Insights and Tools," 
four chapters address some of these organizational realities. Chapter 5 talks about "sludge" - small aspects of an organizationally created context that create impedance for end-users. If sludge is not cleared, the effectiveness of behavioral interventions will be constrained, and hence this chapter makes a case for identifying and eliminating sludge. In chapter 6, Duncan and colleagues provide a guide to writing guidelines, an important tool for most policymakers and businesses as they attempt to provide helpful information to their citizens and customers. Given that organizations have multiple interactions for multiple products and services with their endusers, a binary classification into econs and humans is not feasible or helpful. Therefore, in chapter 7, Ireland talks about the boundedly rational complex consumer continuum, a nuanced framework for segmenting recipients of behavioral interventions. Given that endusers are inundated with information and other types of stimulus from organizations, it is unclear that they will attend to it all. In chapter 8 , Hilchey and Taylor write about the psychology of attention and its implications for helping end-users make better decisions.

Part 3, "Examples of Behavioral Initiatives from Business and Policy," captures results from case studies in several domains of both welfare and business. In chapter 9, Murray and Chen discuss the science of habits and how to change them. In chapter 10, Howe and colleagues describe applications of behavioral science to financial decision making, while in chapter 11, Hardy and colleagues describe a series of projects applying choice-architecture interventions to program development and policy in the Canadian federal government. In chapter 12, Robson discusses how we can use insights to help low-income Canadians file taxes and hence help them obtain many income-tested benefits that are tied to tax returns. In chapter 13, Kim and colleagues present a behavioral approach for studying and tackling issues related to online privacy. In chapter 14, Dalton and colleagues from the World Bank present several cases illustrating the application of BI to international development efforts.

Part 4, "Making It Work," presents two chapters that start identifying key elements and provide guidelines to the practitioner for developing behavioral interventions. In chapter 15, Audet and colleagues discuss the importance of building partnerships and using 
BI in practice. Finally, in chapter 16, Yeung and Tham leverage their experience in working together on several policy issues in the Singapore government, and present a roadmap to help both academics and practitioners navigate the challenges of being scientific in solving practical problems.

For our partnership, this book is merely the beginning of a long, complex, yet important journey. We are confident that the reader will finish this book feeling that they have added an idea or two to their understanding of how to make BI relevant. It will help academics think differently about how to create knowledge that can have an impact in the real world, and it will help practitioners better understand how to read, internalize, and embed science in order to make organizations as behaviorally informed as they could be.

Happy reading.

Dilip Soman, Toronto Catherine Yeung, Hong Kong 